\title{
Memory for a stimulus versus anticipation of a response: Contrasting effects of proactive interference in two delayed comparison tasks
}

\author{
MICHAEL J. PONTECORVO \\ New York University Medical Center, New York, New York
}

\begin{abstract}
Pigeons were trained to perform two delayed comparison tasks that differed only in the temporal placement of the retention interval relative to the sample and comparison stimuli. In one task (conditional delayed response-CDR), the subject could determine the correct response prior to the retention interval. In the second task (delayed conditional response-DCR), the subject was required to compare stimuli presented before and after the retention interval. Although overt mediational responding was not observed in the CDR condition, retention was, nevertheless, greater than in the DCR condition. Furthermore, this difference was amplified at short intertrial intervals. Finally, retention in the DCR, but not the CDR, condition was disrupted by pro. active interference (PI) from previous sample stimuli. The results suggest that memory is less closely tied to the sample stimulus when the stimuli necessary to determine the correct response are presented prior to the retention interval (CDR and other delayed response tasks) than when the subject must compare stimuli across the retention interval (DCR, or delayed matching-tosample, tasks). This difference may lead directly, or indirectly through an interaction with PI, to task differences in retention.
\end{abstract}

Important differences may exist between short-term memory tasks in which the correct response can be determined solely from information presented prior to the retention interval [delayed response tasks (DR), e.g., Carr, 1917; Heise, 1975; Hunter, 1913; Medin, 1969] and tasks that require the subject to compare stimuli presented before and after the retention interval (delayed matching to sample or delayed conditional response (DCR) tasks, e.g., Blough, 1959; D’Amato, 1973; Konorski, 1959; Pontecorvo, 1983). In a DR task, subjects can minimize the memory load by orienting to the correct response alternative during the retention interval (Fletcher, 1965; Hunter, 1913). Thus, the primary role of short-term memory in DR tasks may be to maintain control over the orienting response. Such overt mediational strategies are not possible in DCR tasks, however, because the subject

This research was supported in part by Training Grant ES-07065 and Center Grant ES-00260, both from the National Institute of Environmental Health Sciences, and by Grant OH-00973 from the National Institute of Occupational Safety and Health, U.S. Department of Health and Human Services. The author thanks Hugh Evans for advice and support during the conduct of this research. Raymond Bartus and the American Cyanamid Co. for support and encouragement during preparation of this manuscript. Mary Wilfred for aid in the preparation of the figures, and Charlene Kirby for typing the final draft. George Heise's comments on a preliminary draft are also appreciated, as they greatly improved the focus and clarity of this manuscript. A preliminary report of these results was presented at the meeting of the Eastern Psychological Association, April 1983. Reprint requests should be addressed to M. J. Pontecorvo, Nova Pharmaceutical Corporation. 5210 Eastern Avenue, Baltimore, MD 21224-2788. cannot determine the correct response alternative until after the comparison stimuli have been presented. Thus, more complex memory processes may be required in DCR than in DR tasks.

These differences in memory processes may exist even in situations in which overt mediational responses do not, or cannot, occur; animals in DR tasks may substitute covert coding or mediational strategies for overt orienting. Thus, Konorski $(1959,1961)$ argued that DCR tasks test memory for stimuli, whereas DR tasks test memory for, or anticipation of, responses. More recently, it has been suggested that subjects in DCR tasks learn to anticipate responding to the correct comparison (postdelay) stimulus, rather than remembering the sample (predelay) stimulus (Grant, 1981; Roitblat, 1980). Thus, memory in DR and DCR tasks may be similar, in that anticipatory or "prospective" coding (Grant, 1981; Honig, 1978) may occur in both types of tasks. However, as Honig and Dodd (1983) have pointed out, the content of the memory would still differ along the lines suggested by Konorski. Subjects in a DCR, but not a DR, task must maintain a representation of some stimulus, be it the sample or correct comparison, during the retention interval.

Surprisingly, there have been few empirical comparisons of performance in DR and DCR tasks. Literature reviews suggest that retention may be better in DR than in DCR tests, and that DCR tasks may be relatively more susceptible to interference from previous events (Honig, 1978; Medin \& Davis, 1974; Spear, 1978). However, these conclusions must be qualified, since they are based 
largely on comparisons between studies and, thus, are confounded by numerous procedural differences. A few early studies (Nissen, Riesen, \& Nowlis, 1938; Yerkes \& Yerkes, 1928) did attempt to compare retention in a standard DR task with retention in a "nonspatial" DR task (the equivalent of a DCR task) in which the location of the correct (baited) and incorrect stimulus objects were changed during the retention interval. Although these early studies suggested that retention might be greater in DR than in nonspatial DR tasks, these results are difficult to interpret, because the subjects had histories of responding to spatial location prior to exposure to the test (nonspatial) condition. Furthermore, as Finch (1942) subsequently showed, retention in nonspatial DR tasks may be impaired not only in comparison with that in DR tasks but also in comparison with that in DCR tasks (e.g., delayed matching to sample). Thus, these early studies did not provide an adequate assessment of memory performance/processes in DR and DCR tasks.

Honig and Wasserman (1981) recently attempted to overcome these problems by comparing the performance of pigeons on DR and DCR tasks for which the stimulus sequence, response topography, reinforcement parameters, and amount of training were equated. Consistent with the results cited above, retention in the DR task exceeded that in the DCR task. However, because Honig and Wasserman's DR task involved a less complex discrimination than did their DCR task (simple vs. compound cues), it was not possible to determine whether the differences in retention should be attributed to differences in discrimination difficulty or to differences in memory and mediational processes.

Honig and Dodd (1983) therefore performed a followup study in which both the DR and the DCR tasks required a compound (conditional) discrimination. Pigeons' responses were reinforced following presentation of two compound stimulus pairs (red-horizontal, green-vertical) and not reinforced following presentation of the remaining stimulus pairs (red-vertical, green-horizontal). The DR task (hereafter CDR, for conditional delayed the response) and the DCR task differed primarily in the temporal placement of the retention interval relative to the components of the stimulus pairs. In the CDR task (Honig and Dodd's "combined cues" condition), both components of the stimulus pair were presented prior to the retention interval. In contrast, in the DCR task (Honig and Dodd's "distributed cues" condition), the components of the stimulus pair were separated by the retention interval. Thus, pigeons in the CDR condition, but not those in the DCR condition, could perform the conditional discrimination, and determine the response/reinforcement outcome, prior to the retention interval.

As was the case in Honig and Wasserman's (1981) study, Honig and Dodd found that retention was greater in the CDR than in the DCR condition. Since the discrimination requirements were identical in these two conditions, they suggested that the memory or coding processes employed might be more efficient in DR than in DCR tasks.
However, Honig and Dodd were unable to specify the nature of the processes involved, or to identify the critical procedural difference between the two tasks. Honig and Dodd's CDR and DCR tasks differed in at least two important ways. First, subjects in the CDR condition, but not those in the DCR condition, could determine the correct response prior to the retention interval. Thus, subjects in the CDR condition, but not those in the DCR condition, may have remembered (anticipated) the correct response rather than the sample stimulus. Second, subjects in the CDR condition, but not those in the DCR condition, could also determine (expect) the occurrence or nonoccurrence of reward prior to the retention interval.

The latter difference is important, since it has been shown that differential expectancies of reward can enhance retention in both DR and DCR tasks (Brodigan \& Peterson, 1976; Delong \& Wasserman, 1981). Furthermore, DR and DCR tasks differ in their potential for formation of differential reward expectancies only under limited conditions - that is, when a go/no-go test procedure is employed, and correct nonresponses are not reinforced, as in Honig and Dodd's study. Thus, it is possible that the differences in retention seen in Honig and Dodd's study were not due to intrinsic differences in memory processes between DR and DCR tasks (e.g., Konorski, 1959). Rather, the differences in retention may have been due to a confounding factor-the use of a go/no-go procedure that allowed for the formation of differential reward expectancies in the CDR, but not the DCR, condition.

The present study was intended to provide an unconfounded comparison of retention in CDR and DCR tasks, and to provide more direct evidence concerning the nature of the memory/coding processes employed. The design (Figure 1) was similar to that in Honig and Dodd's study, except that a two-choice delayed comparison procedure (Sahgal \& Iverson, 1978; Shimp \& Moffitt, 1977) replaced Honig and Dodd's go/no-go test procedure. Thus, in both the CDR and DCR conditions, a response on the right key was reinforced if the comparison hue matched the sample hue, whereas a response on the left key was reinforced if the comparison hue did not match the sample. As in Honig and Dodd's study, the CDR and DCR conditions differed only in the placement of the retention interval relative to the sample and comparison stimuli. In the DCR condition, the retention (delay) interval separated the sample from the comparison (conditional) stimulus. Thus, the acronym DCR reflects the order of trial events (delay, conditional stimulus, response). In the CDR condition, the delay interval separated the conditional stimulus from the response opportunity. Thus, the acronym CDR also reflects the order of trial events (conditional stimulus, delay, response).

As in Honig and Dodd's study, subjects in the CDR condition, but not those in the DCR condition, could determine the correct response prior to the retention interval. However, in contrast to Honig and Dodd's study, subjects in the present CDR condition could not form differential expectancies of reinforcement on match and 


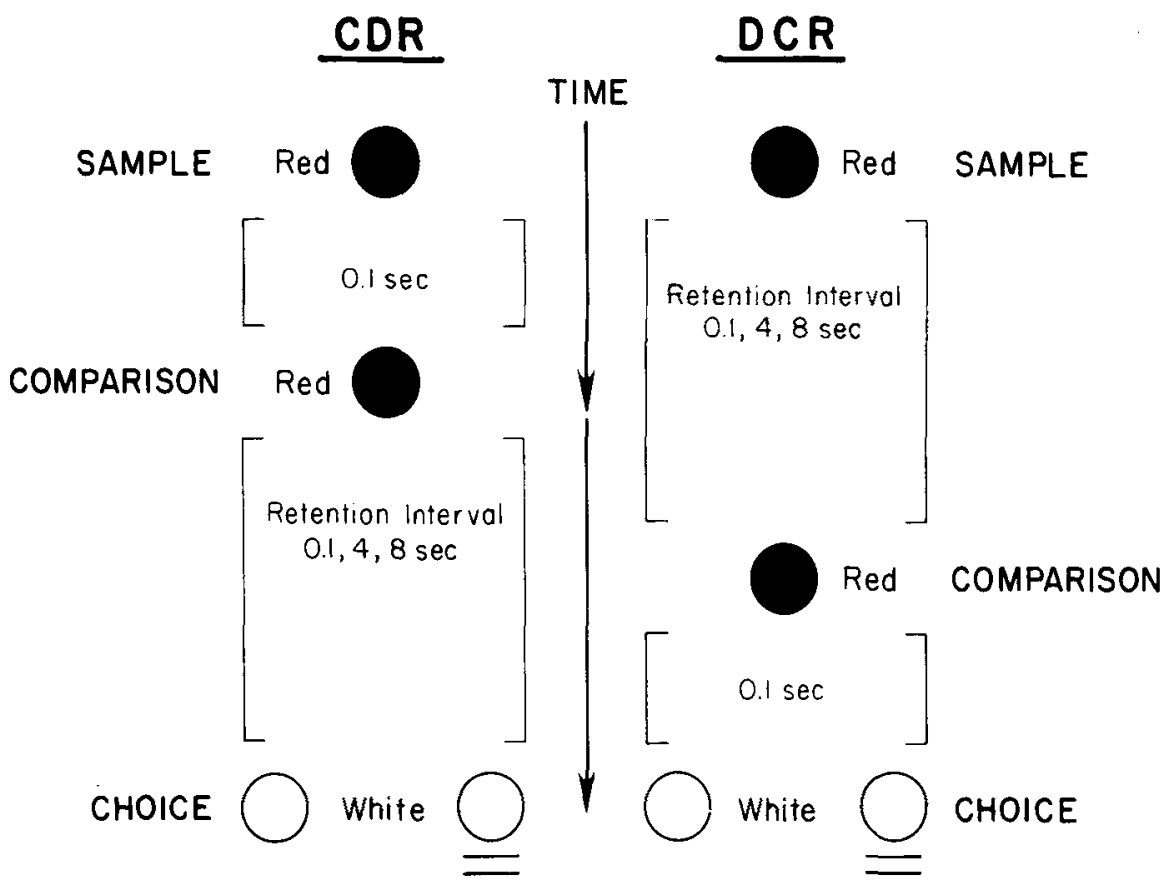

Figure 1. Schematic representation of the procedure used in the present study. For both the CDR and DCR conditions, a sample and comparison (conditional) stimulus were presented successively on the center of three keys, followed by an opportunity to choose one of the two side keys. If the sample matched the comparison, then a response on the right key was reinforced (underlined); otherwise, a response on the left key was reinforced. The DCR and CDR conditions differed only in the placement of the retention interval relative to the sample and comparison stimuli (as shown). In the DCR condition, the retention interval $(0,4,8 \mathrm{sec})$ intervened between the sample and comparison stimuli. In the CDR condition, the retention interval intervened between the conditional stimulus and the response opportunity. Subjects in the CDR condition, but not those in the DCR condition, could determine, prior to the retention interval, which response would be reinforced.

nonmatch trials; the reinforcer could be obtained on every trial, following a correct response. Thus, differences in retention between the present CDR and DCR conditions would clearly suggest that different memory/coding processes are employed in DCR and DR tasks.

Specifically, this result would be consistent with the suggestion (e.g., Konorski, 1959, 1961) that subjects in DCR tasks maintain a representation of some stimulus, whereas subjects in DR tasks remember, or anticipate, the correct response.

This possibility was further explored by examination of retention in the DCR and CDR tasks on a trial-by-trial basis. If subjects in DCR tasks maintain some representation of the sample stimulus, whereas subjects in DR tasks anticipate the opportunity to perform the correct response, then subjects in the DCR task, but not those in the CDR task, should be sensitive to proactive interference (PI) from previous conflicting sample stimuli. That is, memory for previous samples should be disruptive only in the DCR task, in which sample memory is important for retention. Thus, accuracy in the DCR task, but not in the CDR task, should be lower on trials in which the sample stimulus differs from the previous trial's sample than on trials in which the sample is the same as the previous trial's sample. Furthermore, the magnitude of this effect should in- crease with retention-interval duration. Similarly, anticipation of the correct response in the CDR task should be evidenced by a selective vulnerability to PI from previous conflicting response choices. That is, accuracy in the CDR task should be lower on trials in which the correct response differs from the previous trial's correct response than on trials in which the correct response is the same as the previous trial's correct response. Since the magnitude of these PI effects may vary with the intertrial-interval (ITI) duration (see Roberts \& Grant, 1976, and Spear, 1978, for reviews), retention in the CDR and DCR tasks was contrasted at three different ITIs-1, 3, and $12 \mathrm{sec}$.

Finally, the present study examined the pattern of responding on the choice keys during the retention intervals in order to determine the importance of overt mediational responding in the CDR task. If these subjects "bridged" the retention interval by orienting or responding to the to-be-correct choice key (e.g., Fletcher, 1965), then frequent and accurate responding on the choice keys should be observed during the retention interval. Alternatively, if these subjects utilized more covert, cognitive mediational strategies (e.g., Honig, 1978; Konorski, 1959,1961 ), then delay-interval responding would be unnecessary, and thus be less frequent and/or less accurate than choice responding. 


\section{METHOD}

\section{Subjects}

Eight adult male White Carneaux pigeons (Columba livia; Palmetto Pigeon Plant, Sumter, SC) were individually housed in a room with an automatically timed 12-h light-dark cycle. Prior to the start of the experiment, the pigeons were food deprived to $80 \%$ of their ad-lib weights. Thereafter, most birds obtained all of their daily food ration during the experimental session. The subjects received free access to water at all times in the home cage, and supplemental feeding (Purina Pigeon Checkers) was given, as needed, to maintain $80 \%$ weight. The study was run in two replications of 4 birds each. A total of 4 pigeons ( 2 per replication) were tested in each of the CDR and DCR conditions.

\section{Apparatus}

Four identical operant conditioning chambers (No. E10-10, Coulbourn Instruments Inc., Lehigh Valley, PA) were enclosed in lightproof and sound-attenuating cubicles. White noise was presented through a speaker in the ceiling of the chamber. A houselight, located near the floor on the front wall of the chamber, provided diffuse illumination throughout the session, except for a 3.0-sec period following an incorrect response.

Three 2.6-cm-diam stimulus-response keys (Coulbourn No. 2118) were centered horizontally, $8.2 \mathrm{~cm}$ apart (center to center), $25.2 \mathrm{~cm}$ above the floor on the front wall of the chamber. All three keys could be illuminated from the rear with white, red, or green light. A force of approximately $13 \mathrm{~g}$ was required to depress the keys and register a response. A food hopper located below the center key provided $3.0 \mathrm{sec}$ access to mixed grain following each correct response. The apparatus was controlled and the data were recorded by a PDP-8A computer (Digital Equipment Corp., Maynard, MA) with SKED interface (State Systems, Inc., Kalamazoo, MI).

\section{Procedure}

The pigeons were autoshaped to keypeck, then trained to perform a two-choice delayed comparison task (Figure 1). A "sample" stimulus (red or green light) was presented on the center key, followed by a "conditional" or "comparison" stimulus on the same key, and then by a response opportunity, which was signaled by white lights on the two side keys. If the hue of the comparison stimulus matched that of the sample stimulus (match trial), then a peck at the right key was reinforced ( $3 \mathrm{sec}$ access to grain). If the sample and comparison stimuli did not match (nonmatch trial), a left key response was reinforced. An incorrect choice (or no choice) was followed by a 3-sec blackout and then by a repetition of the same sample-comparison sequence (correction trial). A maximum of five consecutive correction trials were permitted. Only data from initial (noncorrection) trials were recorded for further analysis.

The sample stimulus duration was $5 \mathrm{sec}$. The comparison stimulus was presented for a maximum of $3 \mathrm{sec}$ or until five responses were recorded on the center key. The choice stimuli were presented for $3 \mathrm{sec}$ or until a choice response occurred. The intertrial interval (ITI) was $12 \mathrm{sec}$. The interval between the sample and comparison stimulus and the interval between the comparison and choice stimuli were both set at $0.1 \mathrm{sec}$ during the initial stage of acquisition. After the pigeons had achieved a criterion accuracy $\left(\mathrm{A}^{\prime} \geq .90\right.$, averaged over 3 days) or completed a maximum of 25 training sessions, they were alternately assigned to either the DCR or CDR condition and longer retention intervals were gradually introduced. The DCR or CDR conditions differed only in the placement of the retention interval relative to the sample and comparison stimulus. In the DCR condition, the interval between the sample and comparison stimuli was designated the retention interval, and varied (after 5-15 days of preliminary training with shorter intervals) among the values 0.1 (hereafter 0 ), 4 , and $8 \mathrm{sec}$. The interval be- tween the comparison and choice stimuli was fixed at $0.1(0) \mathrm{sec}$. For the CDR task, the interval between the comparison and choice stimuli was designated the retention interval and varied from 0 to $8 \mathrm{sec}$ and the sample-comparison interval was fixed at $0 \mathrm{sec}$.

After approximately 28 sessions of training on the multipleretention-interval CDR or DCR tasks (approximately 50 sessions after the start of the experiment), an 8-session test period was begun. Immediately thereafter, the ITI was decreased from 12 to $1 \mathrm{sec}$ and a second 8-session test period was begun. Finally, the ITI was increased from 1 to $3 \mathrm{sec}$ and a final 8-session test was performed.

\section{Data Analysis}

Since the pigeons typically responded on more than $95 \%$ of all trials, and since the probability of a choice response did not vary across experimental conditions, the few trials in which no choice occurred were excluded from the analysis. Given this exclusion, the data conformed to the model for a yes/no signal detection task (Green \& Swets, 1966). In both the CDR and DCR procedures, a right-key response indicated a "yes" decision (yes, the comparison matched the sample) and a left-key response indicated a "no" decision. Accordingly, the probability of a correct "yes" response on a match trial $[P(\mathrm{Hit})]$ and the probability of an incorrect "yes" response on a nonmatch trial [false alarm, $\mathrm{P}(\mathrm{FA})$ ] were calculated for each pigeon daily. These values were, in turn, used to derive estimates of response accuracy or sensitivity (A'; Grier, 1971; Pollock \& Norman, 1964), the subjects' ability to discriminate match from nonmatch trials, and response bias ( $\beta^{\prime \prime}$; Grier, 1971; Hodos, 1970 ), the relative tendency to respond on the left or right key independent of the trial type or programmed location of the correct response. $\mathrm{A}^{\prime}$ can vary from 0 to 1.0 , with a value of 0.50 indicating chance responding and 1.0 indicating perfect accuracy. $\beta^{\prime \prime}$ can vary from -1.0 to 1.0 , with negative values indicating a bias toward a yes (match, right-key) response and positive values indicating a bias toward a no (nonmatch, left-key) response. The effects of the experimental conditions (i.e., group, retention interval, ITI, and PI) on sensitivity and bias were evaluated using analyses of variance (Dixon, 1981), as described below, with a probability of $p \leq .05$ required to demonstrate significance.

The pattern of responding on the two choice keys during the 4and 8-sec retention intervals of the CDR task was also examined. The total number of responses on each key was recorded separately for match and nonmatch trials. Discrimination ratios analogous to $\mathrm{P}(\mathrm{Hit})$ and $\mathrm{P}(\mathrm{FA})$ were then calculated by dividing the number of responses on the right key by the total number of responses during the retention intervals. In addition, the number of match and nonmatch trial retention intervals in which at least one response occurred on the correct key (e.g., right key during match trials) were also recorded. Thus, it was possible to determine both the distribution of responding during the retention interval and the proportion of trials in which at least one retention-interval response correctly anticipated the appropriate trial response. These values can be compared qualitatively to the corresponding values of $\mathrm{P}(\mathrm{Hit})$, P(FA), and \% correct choice responding.

\section{RESULTS}

Figure 2 shows the mean values of $A^{\prime}$ for the $C D R$ and DCR groups, as a function of ITI and retention-interval duration. It can be seen that response accuracy decreased, for both groups, as the retention interval increased and the ITI decreased. Analysis of variance, with groups (CDR vs. DCR) as the between-subjects factor and retention interval and ITI duration as within-subjects factors, confirmed significant main effects of retention interval 
$\underline{C D R}$

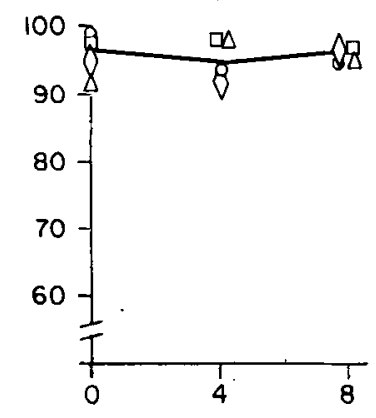

$\underline{\mathrm{DCR}}$
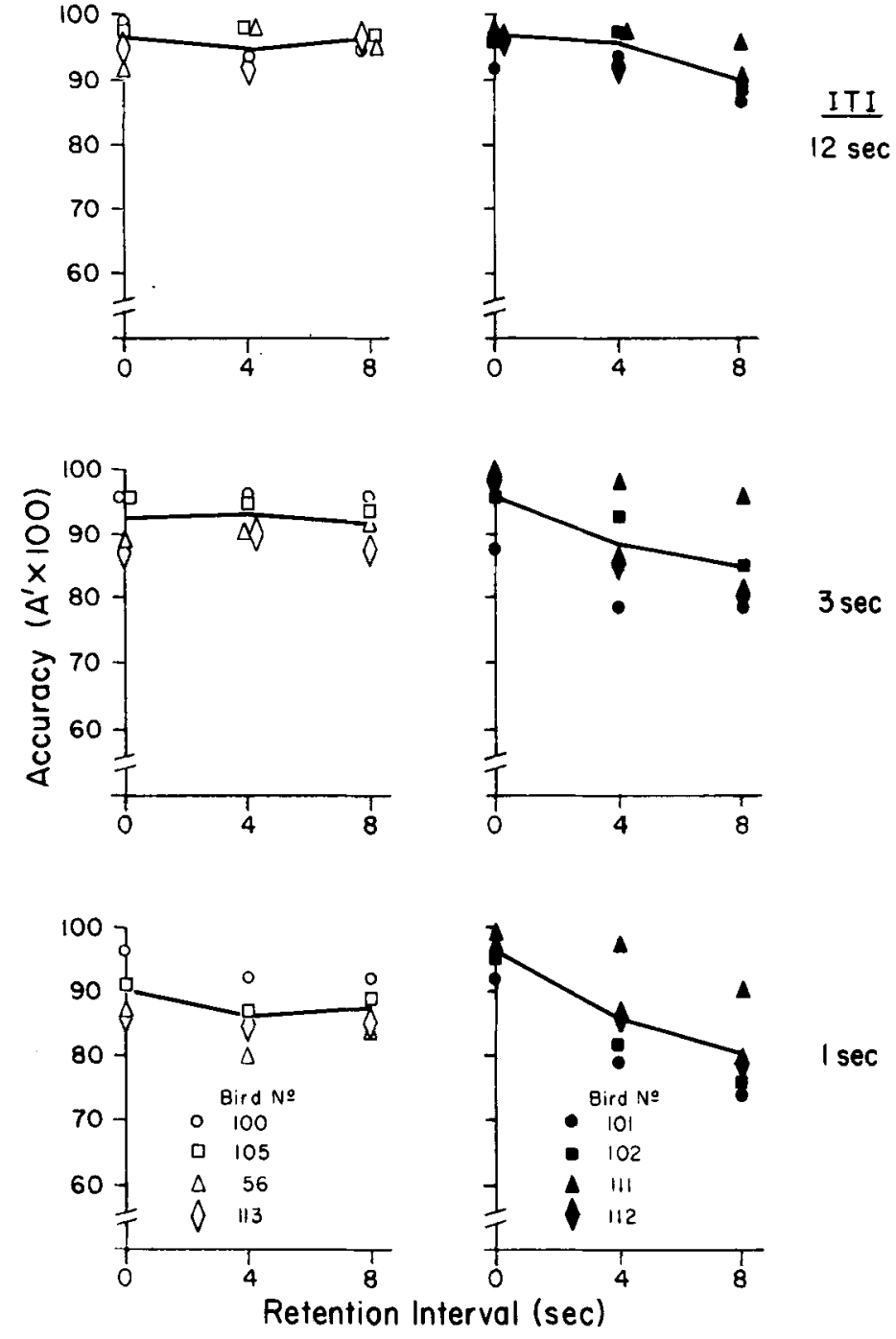

Figure 2. Mean values of $A^{\prime}$ for the $C D R$ and DCR conditions as a function of ITI and retention-interval duration. Points represent values for individual pigeons averaged over an 8-day period. Connected lines represent group means.

$[F(2,12)=23.71]$ and $\operatorname{ITI}[F(2,12)=22.18]$ duration as well as a significant ITI $\times$ retention interval interaction $[F(2,24)=6.83]$. More importantly, there was also a significant groups $\times$ retention interval interaction $[F(2,12)=17.15]$ and a strong trend toward a groups $\times$ retention interval $\times$ ITI interaction $[\mathrm{F}(4,24)=2.68$, $\mathrm{p}<.056]$. Thus, the present results confirmed Honig and Dodd's (1983) finding that retention was significantly greater in the CDR condition than in the DCR condition. Furthermore, the magnitude of this between-groups difference in retention also tended to be greater at short than at long ITI.

The data were further analyzed by computing accuracy $\left(A^{\prime}\right)$ as a function of the relationship between the sample stimulus of the present trial and that of the previous trial and between correct response location of the present trial and that of the previous trial. (Note that, since correction- trial data were not included in this analysis, the previous trial's response was always correct and reinforced.) Figure 3 suggests that subjects in the DCR condition were more sensitive than subjects in the CDR condition to proactive interference (PI) created by trial-to-trial changes in the sample stimulus. Analysis of variance, with groups (CDR vs. DCR) as the between-subjects factor and retention interval, ITI, and sample-stimulus sequence (present sample equals previous trial's sample vs. present sample different from previous trial's sample) as within-subjects factors, confirmed the suggestion. Thus, there was a significant group $\times$ sample sequence interaction $[F(1,6)=$ $6.17]$ and a trend toward a group $\times$ retention interval $\times$ sample sequence interaction $[\mathrm{F}(2,12)=2.81, \mathrm{p}<.10]$, as well as a main effect of sample sequence $[F(1,6)=$ $6.74]$. As in the analysis above, there were also significant main effects for ITI $[F(2,12)=21.54]$ and retention- 
$\underline{\text { CDR }}$
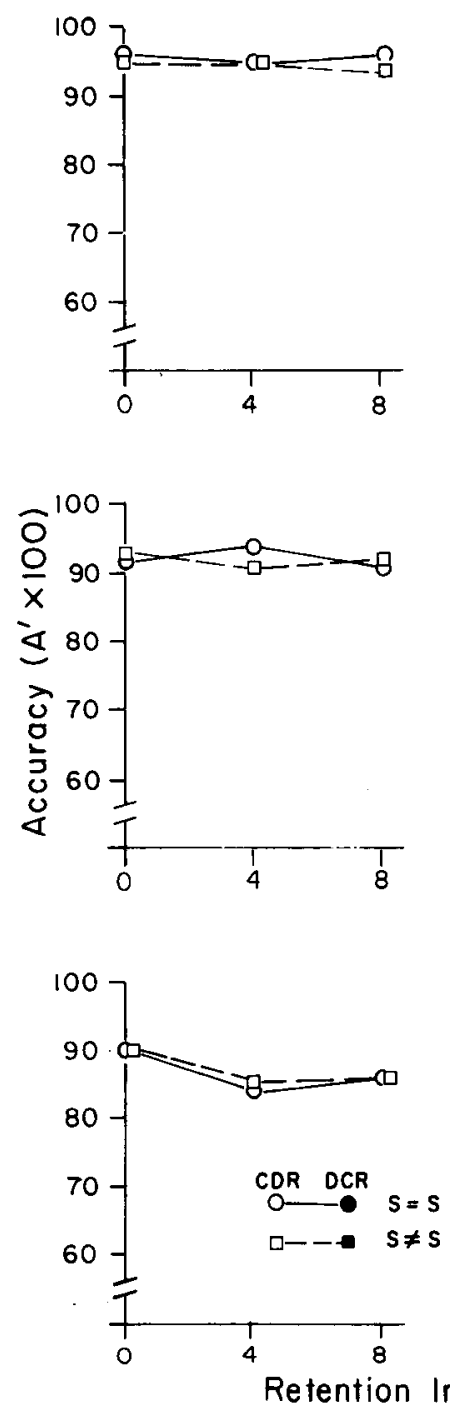

$\underline{D C R}$
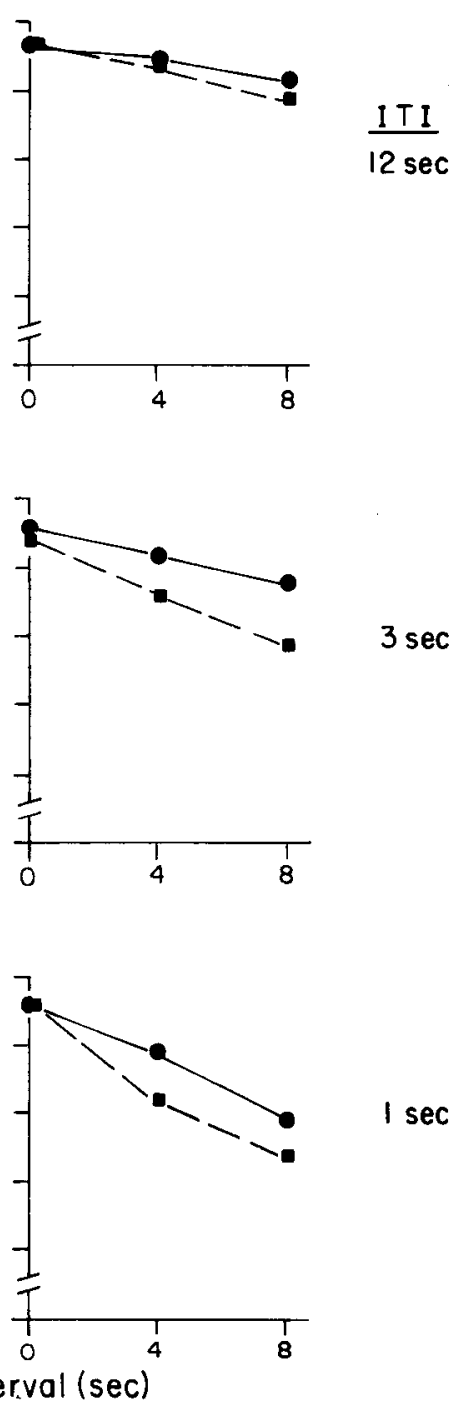

Figure 3. Mean values of $A^{\prime}$ for the $C D R$ and DCR conditions as a function of ITI, retention-interval duration, and relationship between sample stimuli of the present and previous trials. $S=S$ : Present sample same as previous sample (low proactive interference). $S \neq S$ : Present sample different from previous sample (high proactive interference). Values represent group means averaged over each 8-day period.

interval duration $[\mathrm{F}(2,12)=29.34]$, as well as significant ITI $\times$ retention interval $[F(4,24)=7.76]$, group $\times$ retention interval $[F(2,12)=17.04]$, and group $\times$ ITI $X$ retention interval $[F(4,24)=3.14]$ interactions.

In contrast, subjects in both the CDR and DCR conditions were sensitive to PI from trial to trial changes in correct response location (Figure 4). Furthermore, the magnitude of this interference increased as the ITI decreased. Analysis of variance, with groups as the between-subjects factor and retention interval, ITI, and response location (present correct response location same as previous vs. present correct response location different from previous) as within-subjects factors, revealed a significant main effect of response location $[F(1,6)=$ $15.15]$, but no group $\times$ response location interaction
$[F(1,6)=0.07]$. Similarly, there was an ITI $\times$ response location interaction $[F(2,12)=6.75]$ and a strong trend toward a retention interval $\times$ ITI $\times$ response location interaction $[F(4,24)=1.62, p<.061]$, but these trends did not differ between the CDR and DCR groups. Finally, there were significant main effects of ITI $[F(2,12)=$ $19.02]$ and retention-interval duration $[F(2,12)=27.25]$, as well as significant ITI $\times$ retention interval $[\mathrm{F}(4,24)$ $=8.33]$, group $\times$ retention interval $[\mathrm{F}(2,12)=17.44]$, and group $\times$ ITI $\times$ retention interval $[\mathrm{F}(4,24)=2.95]$ interactions, all of which are consistent with the data reported above.

Possible between-group differences in bias $\left(\beta^{\prime \prime}\right)$ were also evaluated using an analysis of variance with groups and intertrial interval as factors. This analysis did not re- 


\section{CDR}
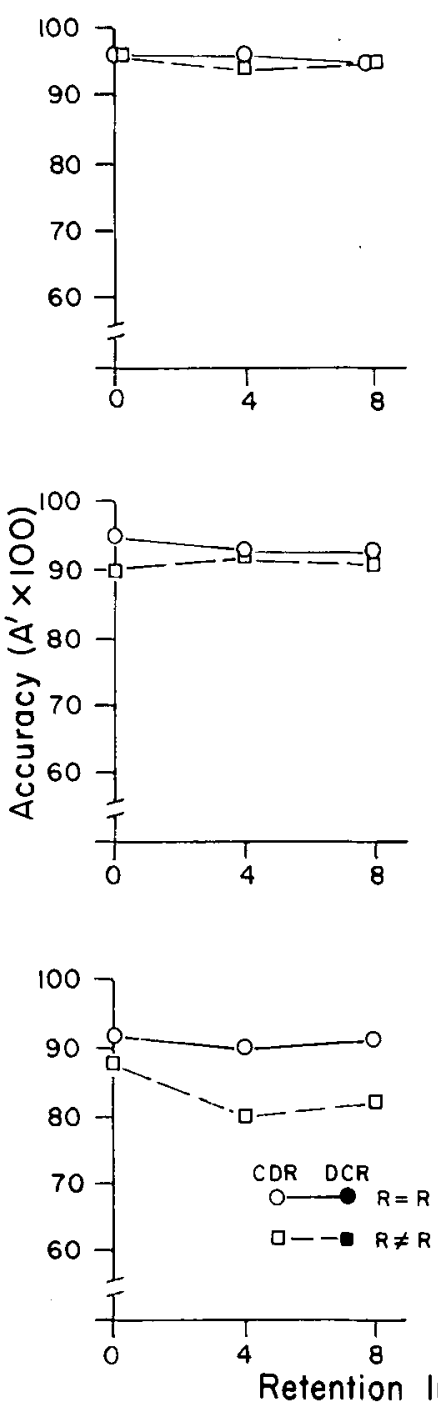

$\underline{D C R}$
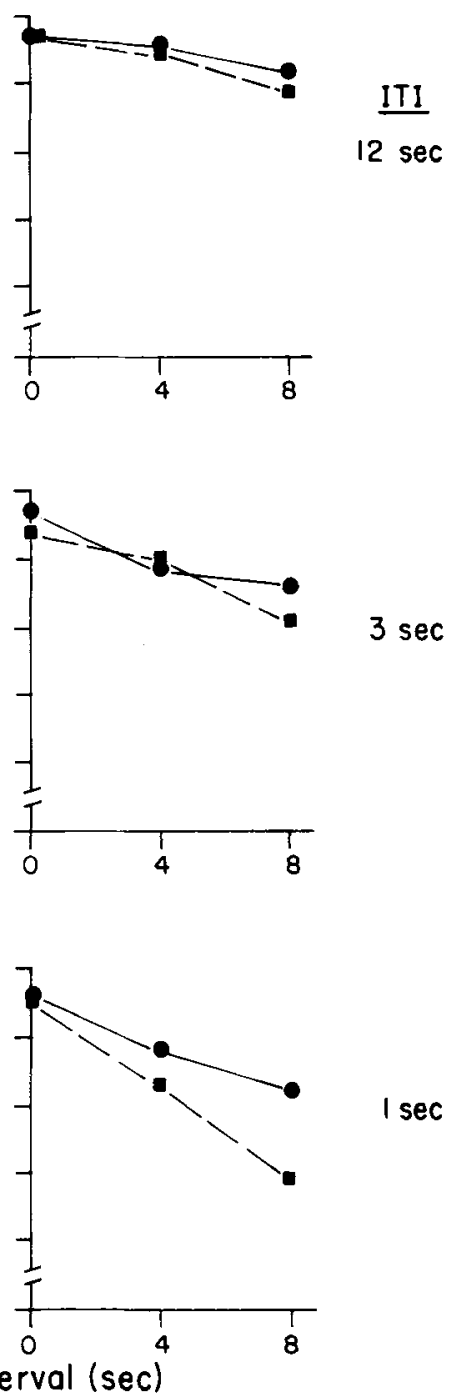

Figure 4. Mean values of $A^{\prime}$ for the $C D R$ and DCR conditions as a function of ITI, retention interval duration, and relationship between the present and previous correct response location. $R=R$ : Present correct location equals previous location (low proactive interference). $R \neq R$ : Present correct location differs from previous location (high proactive interference). As in Figure 3, values represent group means for each 8-day period.

veal significant between-group differences, effects of ITI, or a groups $\times$ ITI interaction.

Finally, examination of the distribution of retentioninterval responses indicated that $99 \%$ of the responses during match-trial and $82 \%$ of the responses during nonmatch-trial retention intervals (averaged across 12-, 3-, and 1-sec ITIs) occurred on the right (match, "yes") key. As shown in Figure 5, these values represent both less accurate responding and a greater bias toward the right key than was seen during the choice periods following the corresponding 4- and 8-sec retention intervals. Similarly, only $52 \%$ of the match-trial and $08 \%$ of the nonmatch-trial retention intervals contained at least one response on the key that would be correct during the choice period. These values also represent less accurate responding, and a greater bias toward the right key than was seen during the choice periods, for which $85 \%$ of the match trials and $82 \%$ of the nonmatch trials contained a correct response.

\section{DISCUSSION}

Although there was no overall difference in response accuracy $\left(A^{\prime}\right)$ between the present $C D R$ and $D C R$ conditions, accuracy decreased more rapidly as a function of retention-interval duration in the DCR condition than in the CDR condition (Figure 2). Thus, these results replicate Honig and Dodd's (1983) finding that retention is 


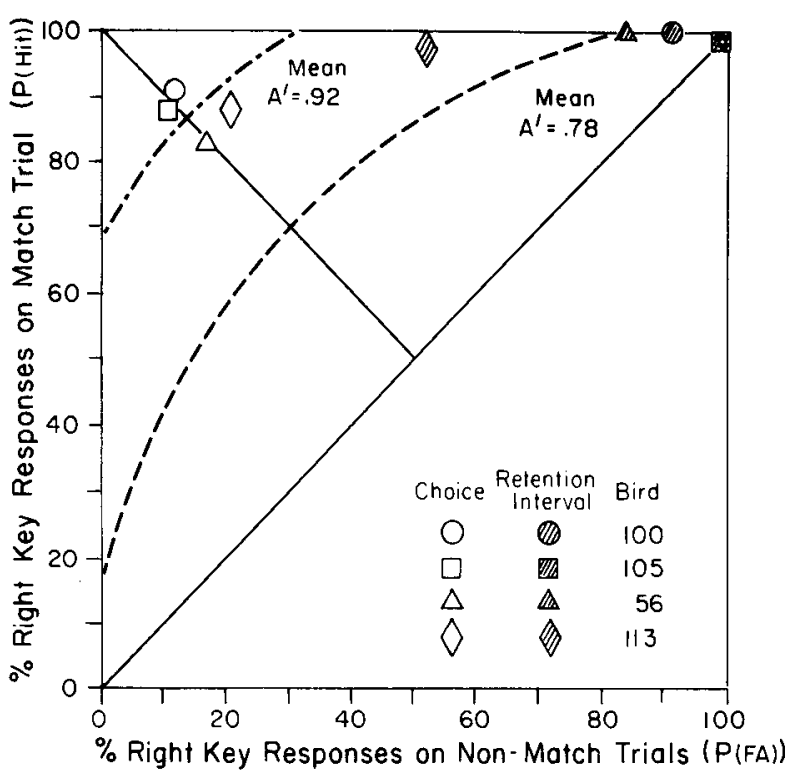

Figure 5. Comparison of responding during the choice periods (open symbols) and retention intervals (shaded symbols) of the CDR condition. Values are averaged across the 4 and 8-sec retentioninterval conditions for all three ITI. Note that accuracy was considerably greater (the points fall farther from the major diagonal) in the choice periods than during the retention intervals.

greater in a CDR than in a DCR procedure. Although the maximum difference in accuracy between the present CDR and DCR conditions was probably not as great as that seen in Honig and Dodd's study, it should be noted that Honig and Dodd's maximal effects were obtained at retention intervals somewhat longer (i.e., 12 and $18 \mathrm{sec}$ ) than those employed in the present study. At retention intervals more comparable to those in the present study (e.g., their 1-, 6-, and 12-sec retention-interval condition), Honig and Dodd's results are remarkably similar to the present results.

Because the present study employed a two-choice test procedure, rather than the go/no-go procedure used by Honig and Dodd, it was not possible for subjects in the present CDR condition to form differential expectancies of reward on match and nonmatch trials. Thus, the only difference between the present CDR and DCR tasks is that both of the stimuli necessary to determine the correct response were present prior to the retention interval in the CDR but not in the DCR condition. That a difference in retention between the CDR and DCR conditions was obtained in both Honig and Dodd's and the present study, therefore, clearly suggests that different memory or mediational processes may be employed in CDR and DCR tasks.

One obvious difference between the present CDR and DCR conditions was that subjects in the CDR, but not the DCR, condition may have "bridged" the retention interval by orienting toward or responding on the to-becorrect choice key (e.g., Fletcher, 1965). However, a number of observations suggest that overt mediational responding did not account for the differences in reten- tion between the two conditions. First, responding during the retention intervals of the CDR condition was considerably less frequent and less accurate than was responding during the choice period (Figure 5). Although this result is not surprising, since reinforcement contingencies did not constrain responding during the retention intervals, it is nevertheless significant. This result suggests that responding during the retention interval was too inaccurate to have served as part of a mediational chain.

Similarly, informal observations suggest that bodily orientation did not serve as part of a mediational chain. During retention intervals of both match and nonmatch trials, birds typically either oriented away from the stimulus panel, engaging in some interim behavior (preening, wing flapping), or approached the right choice key. Because the reward contingencies for the two choice keys were not counterbalanced across animals, it was not possible to determine if this apparent preference for the right key was due to the association of the right key with the "match" response, or to some box asymmetry (e.g., the right key was nearer the door and may have received more illumination from the house lamp). In any case, it seems unlikely that these behaviors could account for the greater retention in the CDR condition. Rather, these data suggest that differences in covert (memory) coding processes account for the differences in retention between the CDR and DCR conditions.

This possibility was further examined by contrasting retention in the CDR and DCR conditions as a function of the relationships between the present and previous trial's sample stimulus and between the present and previous trial's correct response location. The data indicated that retention in the DCR, but not the CDR, condition was disrupted by proactive interference (PI) from trialto-trial changes in the sample stimulus (Figure 3).

The lack of effect of PI from previous sample stimuli in the CDR condition cannot be attributed to insensitivity of the task to experimental manipulation of retention, since retention in the CDR condition was disrupted by PI from trial-to-trial changes in the correct response location. Rather, the results are consistent with the hypothesis that subjects in the CDR task perform the conditional discrimination prior to the retention interval, then utilize a prospective code to remember, or anticipate, the appropriate response to be performed (Honig, 1978; Honig \& Dodd, 1983; Konorski, 1959). Since this strategy does not require the subject to remember the sample stimulus (except for $0.1 \mathrm{sec}$ from sample offset to comparison onset), memory for previous conflicting sample stimuli should not interfere with performance. On the other hand, this strategy does require the subject to remember (anticipate) the correct response. Thus, memories for previous responses should disrupt performance.

In contrast, subjects in the DCR condition acted as though they maintained a representation of the sample, or some stimulus-specific response instruction (e.g., red sample: if red comparison respond right) during the delay. Thus, retention in the DCR condition, unlike the CDR 
condition, was disrupted by PI from trial-to-trial changes in the sample stimulus. Futhermore, this differential sensitivity to PI probably accounted for some, but not all, of the difference in retention between the DCR and CDR tasks. Thus, there was a strong trend for the betweentask differences in retention to be greatest under conditions of high PI $(S \neq S$ condition, Figure 3 ; group $\times$ sample sequence $X$ retention interval interaction $\mathrm{p}<.10$ ). However, differences in retention remained evident across all conditions, including those that would be expected to produce minimal PI (12-sec ITI, $\mathrm{S}=\mathrm{S}$ condition). These findings suggest that differences in the memory/encoding process may have contributed directly, as well as indirectly through an interaction with PI, to the differences in retention between the CDR and DCR conditions.

It is interesting that retention in the DCR condition was also disrupted by PI from trial-to-trial changes in the location of the correct response. Thus, subjects in the DCR condition acted as though they also remembered the location of the previous reinforced response, even though it had no relevance for future reinforcement. Furthermore, control by this memory appeared to increase as retention for the present sample stimulus decreased (i.e., PI was greater at long delays).

A similar tendency to repeat the previous reinforced response may also contribute to the effects of trial-to-trial changes in response location in the CDR condition. Thus, the decreased retention following changes in correct response location may be due in part to a perseverative response tendency, rather than to (or in addition to) a specific interference with memory for the correct choice (as suggested above). That negative transfer from previous choices disrupted performance selectively at very short (1-sec) ITIs is consistent with this hypothesis. It is important to note, however, that this would not alter the overall interpretation of the present results. The critical finding in the present study is that retention in the DCR condition, but not in the CDR condition, is disrupted by PI from trial-to-trial changes in the sample stimulus (Figure 3). Thus, memory for the sample stimulus does not appear to be important for retention in the CDR task.

The present results are therefore consistent with the hypothesis (Konorski, 1959) that DCR tasks reflect memory for some stimulus, whereas DR tasks reflect memory for or anticipation of a response. What remains to be determined is which stimulus controls memory in a DCR task. That is, it remains uncertain whether subjects in DCR tasks code retrospectively, maintaining a representation of the sample stimulus (e.g., Roberts \& Grant, 1976), or prospectively, remembering or anticipating the correct comparison stimuli (e.g., Grant, 1981; Roitblat, 1980). Both types of code appear more complex and stimulus dependent than the simple "response instruction" (e.g., respond left) utilized in the CDR task. Thus, both could account for the difference in retention and sensitivity to (stimulus) PI between the present DCR and CDR conditions.
A number of studies have compared retrospective and prospective accounts of DCR performance (see Grant, 1981; Honig, 1978, 1981; Roitblat, 1980). However, a definitive test of these hypotheses has yet to be performed. Furthermore, it should be noted that these alternative memory/coding strategies are not necessarily mutually exclusive. Rather, a continuum may exist along which subjects place more or less emphasis on prospective or retrospective encoding mechanisms. The present analysis suggests that subjects in DCR tasks should tend to fall closer on the continuum toward retrospective, stimulusdependent coding than do subjects in DR (CDR) tasks.

\section{REFERENCES}

BLough, D. S. (1959). Delayed matching in the pigeon. Journal of the Experimental Analysis of Behavior, 2, 151-160.

Brodigan, D. L., \& Peterson, G. B. (1976). Two-choice conditional discrimination performance of pigeons as a function of reward expectancy, prechoice delay, and domesticity. Animal Learning \& Behavior, 4, 121-124.

CARR, A. M. (1917). The alternation problem. Journal of Animal Behavior, 7, 365-384.

D'Aмato, M. R. (1973). Delayed matching and short-term memory in monkeys. In G. H. Bower (Ed.), The psychology of learning and motivation: Advances in research and theory (Vol. 7). New York: Academic Press.

Delong, R. E., Wasserman, E. A. (1981). Effects of differential reinforcement expectancies on successive matching-to-sample performance in pigeons. Journal of Experimental Psychology: Animal Behavior Processes, 7, 394-412.

Dixon, W. J. (1981). BMDP statistical software. Berkeley: University of California Press.

FINCH, G. (1942). Delayed matching-from-sample and non-spatial delayed response in chimpanzees. Journal of Comparative Psychology, 34, 315-319.

Fletcher, H. J. (1965). The delayed-response problem. In A. M. Schrier, H. F. Harlow, \& F. Stollnitz (Eds.), Behavior of nonhuman primates (Vol. 1). New York: Academic Press.

Grant, D. S. (1981). Short-term memory in the pigeon. In N. E. Spear \& R. R. Miller (Eds.), Information processing in animals: Memory mechanisms. Hillsdale, NJ: Erlbaum.

GreEn, D. M., \& SWETS, J. A. (1966). Signal detection theory and psychophysics. New York: Wiley.

GrIER, J. (1971). Nonparametric indexes for sensitivity and bias: Computing formulas. Psychological Bulletin, 75, 424-429.

HEISE, G. A. (1975). Discrete trial analysis of drug action. Federation Proceedings, 34, 1898-1903.

Hodos, W. (1970). Non-parametric index of response bias for use in detection and recognition experiments. Psychological Bulletin, 74, 351-354.

HonIG, W. K. (1978). Studies of working memory in pigeons. In S. H. Hulse, H. Fowler, \& W. K. Honig (Eds.), Cognitive processes in animal behavior. Hillsdale, NJ: Erlbaum.

HoNIG, W. K. (1981). Working memory and the temporal map. In N. E. Spear \& R. R. Miller (Eds.), Information processing in animals: Memory mechanisms. Hillsdale, NJ: Erlbaum.

HoniG, W. K., DoDD, P. W. D. (1983). Delayed discriminations in the pigeon: The role of within-trial location of conditional cues. Animal Learning \& Behavior, 11, 1-9.

Honig, W. K., \& Wasserman, E. A. (1981). Performance of pigeons on delayed simple and conditional discriminations under equivalent training procedures. Learning \& Motivation, 12, 149-170.

HUNTER, W. S. (1913). The delayed reaction in animals and children. Behavior Monographs, 2, 1-86.

KONORSKI, J. A. (1959). A new method of physiological investigation 
of recent memory in animals. Bulletin of the Polish Academy of Sciences, 7, 115-117.

Konorski, J. A. (1961). The physiological approach to the problem of recent memory. In A. Fessard, R. W. Gerard, J. A. Konorski, \& J. F. Delafresnaye (Eds.), Brain mechanisms and learning. Oxford: Blackwell.

MEDIN, D. L. (1969). Form perception and pattern reproduction by monkeys. Journal of Comparative \& Physiological Psychology, 68, 412-419.

Medin, D. L., \& Davis, R. T. (1974). Memory. In A. M. Schrier \& F. Stollnitz (Eds.), Behavior of non-human primates. New York: Academic Press.

NisSEn, H. W., Riesen, A. H., \& Nowlis, V. (1938). Delayed response and discrimination learning by chimpanzees. Journal of Comparative Psychology, 26, 361-386.

Pollock, I., \& Norman, D. A. (1964). A non-parametric analysis of recognition experiments. Psychonomic Science, 1, 125-126.

Ponteconvo, M. J. (1983). Effects of proactive interference on rats' continuous nonmatching-to-sample performance. Animal Learning \& Behavior, 11, 356-366.

RoberTs, W. A., \& Grant, D. S. (1976). Studies of short-term memory in the pigeon using the delayed matching to sample procedure. In D. L. Medin, W. A. Roberts, \& R. T. Davis (Eds.), Processes of animal memory. Hillsdale, NJ: Erlbaum.

RoItblat, H. L. (1980). Codes and processing in pigeon short-term memory. Animal Learning \& Behavior, 8, 341-351.

Sahgal, A., \& Iversen, S. D. (1978). The effects of chlordiazepoxide on a delayed pair comparison task in pigeons. Psychopharmacology, 59, 57-64.

Shimp, C. P., \& MoffitT, M. (1977). Short-term memory in the pigeon: Delayed-pair comparison procedures and some results. Joumal of the Experimental Analysis of Behavior, 28, 13-25.

SPEAR, N. E. (1978). The processing of memories: Forgetting and retention. Hillsdale, NJ: Erlbaum.

YeRKES, R. M., \& YeRKES, D. M. (1928). Concerning memory in the chimpanzee. Journal of Comparative Psychology, 8, 237-271.

(Manuscript received May 3, 1985;

revision accepted for publication September 23, 1985.) 